\title{
ANALYSIS OF THE ACOUSTIC FIELD IN A THERMOACOUSTIC SYSTEM USING TIME- RESOLVED PARTICLE IMAGE VELOCIMETRY AND CONSTANT-VOLTAGE ANEMOMETRY
}

\author{
P. Blanc-Benon ${ }^{1 *}$, E. Jondeau ${ }^{1}$, and G. Comte-Bellot ${ }^{1}$ \\ ${ }^{1}$ Laboratoire de Mécanique des Fluides et d'Acoustique, UMR CNRS 5509, Université de Lyon, École Centrale de Lyon, 36 \\ avenue Guy de Collongue, 69134 Ecully-Cedex, France. \\ . *Corresponding author's e-mail: philippe.blanc-benon@ec-lyon.fr
}

Keywords: nonlinear acoustic, oscillating flows, temperature fluctuation, velocity fluctuation, timeresolved particle image velocimetry, constant-voltage anemomety, thermoacoustic,

The development of high-performance thermoacoustic systems requires efficient heat transfer between the stack or the regenerator and the heat-exchangers. So far, the coupling between these elements has been addressed using theoretical models and numerical simulations ${ }^{1}$. These analyses demonstrate that, at the high acoustic levels found in realistic thermoacoustic systems, acoustic velocities and instantaneous temperatures exhibit nonlinear interactions. However, there is a lack of controlled experimental data to validate these results. In this paper we consider a standing-wave thermoacoustic refrigerator. It consists of a stack of plates placed in an acoustic resonator. Two heat exchangers are located at each stack extremity. The thermoacoustic effect takes place in the thermal and viscous boundary layers along each plate of the stack. It results in a heat transport along the plates and in a temperature difference between the two stack ends. The full understanding of the heat transfer between the stack and the heat exchangers is a key issue to improve the global efficiency of these devices.

The aim of this analysis is to investigate the vortex structures, which appear at the ends of the stack and modify the heat transfer. Here, the aerodynamic in the gap stack-exchanger is characterized using a time-resolved particle image velocimetry technique ${ }^{2}$ (TR-PIV). Measurements are performed in a device operating at a frequency of $200 \mathrm{~Hz}$. Instantaneous velocity fields are recorded at a frequency of $3125 \mathrm{~Hz}$ (ie 15 maps per acoustic period). Measurements show that vortex shedding occur at high pressure levels, when a nonlinear acoustic regime prevails, leading to an additional heating generated by viscous dissipation in the gap and a loss of efficiency.

In order to analyze the structure of the instantaneous temperature field we have developed a new thermal anemometry technique using a constant-voltage anemometer (CVA) prototype ${ }^{3,4,5}$. This technique is well adapted to unsteady and oscillating flows as the thermal lag of the sensors is corrected instantaneously. It is based on an additional measurement of the flow velocity and its influence on the heat transfer balance of the sensor. Using specifically designed probes with very fine wires, high frequency signals can be recorded and analysed. In a first part we present a validation of this new procedure using a standing-wave acoustic tube for which the fluctuating temperature field is well known even for high acoustic pressure levels. In a second part, measurements are performed behind the stack of a standing-wave thermoacoustic refrigerator. They confirm the existence of temperature harmonics near the stack edge, which should not be overlooked when designing the stack and the heat exchangers. 
In summary, the TR-PIV gives access to the real time evolution of the flow rather than to rely on phase-averaging and it allows measurements even when the flow loses its periodicity at high drive ratio. The development of a new procedure for the measurement of temperature fluctuations which relies on the unique features of the CVA, allows measurement of temperature fluctuations in oscillating flows. With these two experimental techniques, it is now possible to have an estimation of the nonlinearities which occur in efficient thermoacoustic systems.

\section{References}

[1] Marx, D. and Blanc-Benon, P., "Numerical simulation of stack-heat exchangers coupling in a thermoacoustic refrigerator", AIAA Journal, (2004), 42(7), 1338-1347.

[2] Poignand G., Jondeau E. and Blanc-Benon P., "Investigation of the aerodynamic field in a standing-wave thermoacoustic refrigerator using Time-Resolved Particle Image Velocimetry", 17th AIAA/CEAS Aeroacoustics Conference, (2011),AIAA Paper 2011-2932.

[3] Berson, A., Poignand, G., Blanc-Benon P. and Comte-Bellot, G., “Nonlinear temperature field near the stack ends of a standing-wave thermoacoustic refrigerator", Int. J. Heat Mass Transfer, (2011),54(21-22), 4730-4735

[4] Berson A., Poignand G., Blanc-Benon P. and Comte-Bellot G., "Capture of instantaneous temperature in oscillating flows: Use of constant-voltage anemometry to correct the thermal lag of cold wires operated by constant-current anemometry", Review of Scientific Instruments, (2010) 81(1), 015102.

[5] Taifour A. M., Weiss J., Sadeghi A., Vétel J., Jondeau E. and Comte-Bellot G., “A detailed procedure for measuring turbulent velocity fluctuations using constant voltage anemometry", Experiment in Fluids (2015) 56:17, DOI 10.1007/s00348-015-2045-0. 